\title{
Irradiation of UV-B Induces Biosynthesis of Anthocyanins in Flower Petals of Rose, Rosa hybrida cv. 'Charleston' and 'Ehigasa'
}

\author{
Chandani Kumari HennayaKe, Michio KANEChI, Nanako YaSUdA*, Yuichi Uno* \\ and Noboru INAGAKI \\ Graduate School of Science and Technology, Kobe University, Kobe, Hyogo 657-8501, Japan \\ * Faculty of Agriculture, Kobe University, Kobe, Hyogo 657-8501, Japan
}

(Received September 9, 2005)

\begin{abstract}
The ultraviolet-B radiation (UV-B: 280 to $320 \mathrm{~nm}$ ) promotes the accumulation of the anthocyanins in rose plants. Open flowers of Rosa hybrida cultivars 'Charleston' and 'Ehigasa' undergo a striking color change from yellow to red over 10-12 days under natural daylight due to the accumulation of the anthocyanins in the petals. The anthocyanin present in the rose petals was identified as cyanidin 3-glucoside (chrysanthemin) and cyanidin 3,5-diglucoside (cyanin) by using paper chromatography, partial acid hydrolysis, and HPLC. To determine the role of UV-B in the anthocyanin biosynthesis pathway, rose plants were grown under different light conditions supplemented with or without UV-B. Anthocyanin accumulation was suppressed in the treatment without UV-B and the accumulation started following to exposure to UV-B by a gradual increase of cyanin, followed by a dramatically increase of chrysanthemin. Furthermore, it revealed that the unfolding flower stage 3 to 4 (about $50 \%$ anthesis) was critical for anthocyanin biosynthesis in rose.
\end{abstract}

Keywords : anthocyanin, chrysanthemin, cyanin, Rosa hybrida, ultraviolet radiation

\section{INTRODUCTION}

In recent years global-scale environmental destruction has been going on, and the depletion of the stratospheric ozone layer is one of the key issues to be concerned about. It is well known fact that the depletion of the ozone layer is increasing the level of ultraviolet-B radiation (UV-B: $280^{-}$ $320 \mathrm{~nm}$ ) reaching to the surface of the earth (Kerr and McElroy, 1993). In general, UV-B radiation has been shown to be harmful to living organisms, damaging DNA, proteins, and lipids (Kochevar, 1990; Jordan, 1996). A major effect of UV-B radiation on plants is the declination of the plant growth and development and ultimately the decreases in the yield. The mechanisms by which plants may protect themselves from UV-B radiation are interest. One important defense mechanism appears to be the accumulation of the anthocyanins in the cell absorbing UV-B radiation (Beggs et al., 1986) and UV-B radiation is one of the major environmental factors influencing anthocyanin accumulation in flowers. It has been reported that UV-B radiation regulates the anthocyanin biosynthesis in some other plant species (Hashimoto et al., 1984; Arakawa et al., 1985; Yatsuhashi and Hashimoto, 1985; Kolb et al., 2001; Yoshida et al., 2003). However, UV-A (320-

Corresponding author: Michio Kanechi, fax : +81-78-803-5831

e-mail : kanechi@kobe-u.ac.jp 


\section{K. HENNAYAKE ET AL.}

$380 \mathrm{~nm}$ ) is not attenuated by atmospheric ozone, and this less damaging radiation is an important photomorphogenic signal in plant development and photorepair (Björn, 1994; Allen et al., 1999) and it has been reported that UV-A radiation caused an accumulation of flavonoids, which has frequently been suggested in the literature (Robberecht and Caldwell, 1983; Hirner et al., 2001; Lavola et al., 2003).

Study of anthocyanins today is undoubtedly one of the emerging areas of intense research activity and has tremendous potential in agriculture, horticulture and also pharmaceutical industry. Therefore, there is possibility of UV-B radiation reaching the earth can be practically used in the field of agriculture such as, flower breeding research and modulators of hormone response. The flower pigments, mainly important to attract pollinators, have been well characterized. Anthocyanins are major flower pigments in higher plants that are important to protect themselves against microbial attack, herbivores and UV radiation, and have also pharmacological activities in mammals and are used to treat different diseases (Li et al., 1993; Gantet and Memelink, 2002). Scientists are now discovering the importance of anthocyanins and beginning to appreciate their health benefits.

For centuries, rose has been the most important cut flower in the floriculture industry worldwide. The rose petals are known to synthesize pelargonidin, cyanidin and peonidin type anthocyanins (Biolley et al., 1994). In spite of the extensive knowledge of the effect of UV radiation on the biosynthesis of anthocyanins, very little information has been reported for rose. Therefore, study of anthocyanin biosynthesis by UV radiation in rose is important and can be used to improve the quality of flower color because of the commercial value of the rose.

Here, we used Rosa hybrida cultivars 'Charleston', a climbing type, originated in Australia and 'Ehigasa', a floribunda type, originated in Japan that open flowers undergo a striking color change from yellow to red over 10-12 days under natural daylight. This color change is due to the accumulation of the anthocyanins in the petal, and therefore both cultivars are suitable materials among 24,000 cultivars of roses for the research of anthocyanin biosynthesis. In the present study, we researched the content of anthocyanins and the effect of UV-B radiation on anthocyanin biosynthesis by exposing the bud or flower to different light conditions in both cultivars.

\section{MATERIALS AND METHODS}

\section{Plant materials}

Petals of Rosa hybrida cultivars 'Charleston' and 'Ehigasa' were obtained during spring season from the experimental field and greenhouse of Faculty of Agriculture, Kobe University. Plants were grown under standard cultural practices and maintained in a greenhouse under natural photoperiod of 12 to $13 \mathrm{~h}$ at approximately $26 \pm 2^{\circ} \mathrm{C}$ of air temperature. Five different developmental stages of flowers (Fig. 2) under different treatment conditions were harvested into liquid nitrogen and stored at $-20^{\circ} \mathrm{C}$ until use.

\section{$U V$ irradiation and the light switching treatment}

The greenhouse-grown plants were supplemented with either UV-B or UV-A radiation by standard UV-B fluorescent tubes (FL 20SE 20W, Toshiba, Japan) and UV-A fluorescent tubes (FL 20SBL-B, NEC, Japan), respectively. In 'light switching' experiments, six types of UV-cut treatment were designed for field grown plants by covering each bud or flower with UV-cut film (Murasaki-A, Norbi, Mitsubishi, Japan) during various unfolding stages and named as follows: treatment $\mathrm{A}$, flowers were covered through the stage 1-3; treatment $\mathrm{B}$, flowers were covered through the stage 1-4; treatment $C$, flowers were covered through the stage 3-5; treatment $D$, flowers were covered through the stage 4-5; treatment $\mathrm{E}$ (negative control), flowers were covered through all stages; treatment $F$ (positive control), flowers never covered and exposed to natural UV radiation through all stages. Absorption spectra in all treatment were measured with a 


\section{ANTHOCYANIN BIOSYNTHESIS IN ROSE}

spectroradiometer (SR 9910, Macam Photometrics, UK). For each treatment, unfolded petals were harvested at stage 5 , immediately frozen and stored at $-20^{\circ} \mathrm{C}$ until use.

\section{Extraction and quantification of the anthocyanins}

Rose petals from each stage ( $0.5 \mathrm{~g}$ fresh weight) were ground to a fine suspension in $2 \mathrm{ml}$ of acetic acid:methanol $(1: 1)$ solution in a small glass homogenizer. Then, the extracts were centrifuged at $14,000 \mathrm{rpm}$ for $5 \mathrm{~min}$ at $20^{\circ} \mathrm{C}$. The supernatant was filtered through a micro-filter $(0.45$ $\mu \mathrm{m}$ DISMIC-13HP, Toyo Roshi, Japan) prior to HPLC analysis. Two methods of quantitative analysis were performed. The first, classical techniques such as paper chromatography and partial acid hydrolysis analysis were used and secondly HPLC coupled with photodiode array detection was carried out for more precise analysis.

In paper chromatography, a few drops of extracts were separated and determined the rate of migration. After re-extraction of the migrated band, the partial acid hydrolysis with acid methanol was performed and observed the number of spots on the second paper chromatography. HPLC analysis was performed with a HPLC (Waters ${ }^{\mathrm{TM}} 600 \mathrm{E}$ Controller, Milford, USA) system by modifying the procedures of Graham (1991) as follows. Ten micro-liter of the filtrated supernatant was injected into a $\mathrm{C}_{18}$ reversed-phase column (Mightysil RP-18GP (ODS), $5 \mu \mathrm{m}, 250 \times 4.6 \mathrm{~mm}$, Kanto Chemical Co., Japan). The separation of anthocyanins was done by using a 10 min elution of $1.5 \%$ (v/v) phosphoric acid in water, followed by a 20 min linear gradient increase elution from $30 \%$ to $50 \%$ of $1.5 \%(\mathrm{v} / \mathrm{v})$ phosphoric acid and $20 \%(\mathrm{v} / \mathrm{v})$ acetic acid and $25 \%(\mathrm{v} / \mathrm{v})$ acetonitrile in water. The column chromatography was performed at $35^{\circ} \mathrm{C}$ and samples were eluted at a flow rate of 1 $\mathrm{ml} \mathrm{min}{ }^{-1}$. Anthocyanins were monitored at $520 \mathrm{~nm}$ using a Photodiode Array Detector (Waters ${ }^{\mathrm{TM}}$ 996). The identification of anthocyanins and their concentrations were determined by co-injecting cyanidin 3,5-di- $O$-glucoside chloride and cyanidin 3-O-glucoside as standards (Extrasynthese, France).

\section{RESULTS AND DISCUSSION}

As a first step towards determining the type of anthocyanins in rose, anthocyanins were purified from cultivar 'Charleston' and qualitatively analyzed by using paper chromatography, partial acid hydrolysis and HPLC (Table 1). Based on the known spectral characteristics and the hydrolytically released numbers of anthocyanin compounds of the other species (Harborne and Grayer, 1988; Brugliera et al., 1999) and authentic standards, we identified two kinds of anthocyanins namely chrysanthemin (cyanidin 3-glucoside) and cyanin (cyanidin 3,5-diglucoside). Our result was consistent with Shisa and Takano (1964), because they have recognized chrysanthemin and cyanin are the major anthocyanins in related rose cultivars of 'Crimson Glory' and 'Masquerade' which change their petal color from yellow to red during the anthesis.

To investigate the effect of UV radiation, rose plants were grown under different light conditions with or without exposing to UV radiation by covering flowers with UV-cut film. Figure 1 shows the absorption spectra of UV-A, UV-B or without UV radiation measured with a spectroradiometer either in the field or in the greenhouse. The plants that were exposed to the supplemented UV-B radiation $(<320 \mathrm{~nm})$ under greenhouse conditions showed higher absorbance than the absorbance of natural radiation in the greenhouse (Fig. 1, curve A and C, respectively). In contrast to UV-B absorbance, the supplemental UV-A radiation $\left(320^{-}-380 \mathrm{~nm}\right)$ observed slightly increased absorbance compare with natural UV radiation in the greenhouse (Fig. 1, curve B and C, respectively). Although greenhouse conditions restricted receiving a part of UV radiation (300$340 \mathrm{~nm}$ ), field-grown plants received the entire range of natural UV radiation and therefore, it exceeded the absorbance in greenhouse (Fig. 1, curve C and D). This attributes to have higher resolution view of open flowers of field-grown plants that undergo a striking color change from yellow to deep red than the greenhouse flowers under natural daylight. Figure 1, curve $\mathrm{E}$ demonstrates that 


\section{K. HENNAYAKE ET AL.}

Table 1 Qualitative analysis of anthocyanins. Two bands were detected as 'Band A' and 'Band B' with paper chromatography (BtOH: $36 \% \mathrm{HCl}: \mathrm{H}_{2} \mathrm{O}=5: 1: 2$ ).

\begin{tabular}{|c|c|c|c|c|}
\hline & $\begin{array}{l}\text { Chrysanthemin } \\
\text { (Band A) }\end{array}$ & $\begin{array}{l}\text { Cyanin } \\
\text { (Band B) }\end{array}$ & Standard- $1^{2}$ & Standard- $2^{z}$ \\
\hline Rf value & 0.44 & 0.21 & 0.22 & 0.43 \\
\hline Number of spots ${ }^{x}$ & 2 & 4 & 4 & 2 \\
\hline HPLC retention time $(\mathrm{min})$ & 24 & 21 & 21 & 24 \\
\hline Absorbance maximum (nm) & 518.2 & 515.2 & 514.9 & 517 \\
\hline$E_{440} / E_{\max }(\%)^{n}$ & 31.3 & 17.4 & 17.6 & 30.2 \\
\hline
\end{tabular}

' Standard-1: cyanidin 3,5-di-O-glucoside chloride; Standard-2: cyanidin 3-O-glucoside purchased commercially.

"The ratio of distance traveled by substance and distance traveled by solvent front (migration rate).

${ }^{x}$ The number of spots was detected as the result of paper chromatography $\left(\mathrm{BtOH}: 36 \% \mathrm{HCl}: \mathrm{H}_{2} \mathrm{O}=7: 2: 5\right)$ after the time controlled partial acid hydrolysis at $100^{\circ} \mathrm{C}$ in $3 \mathrm{~N} \mathrm{HCl}$ for 10 to $120 \mathrm{~min}$.

"The percentage of absorption coefficient at $440 \mathrm{~nm}$ over absorption coefficient at maximum absorbance.

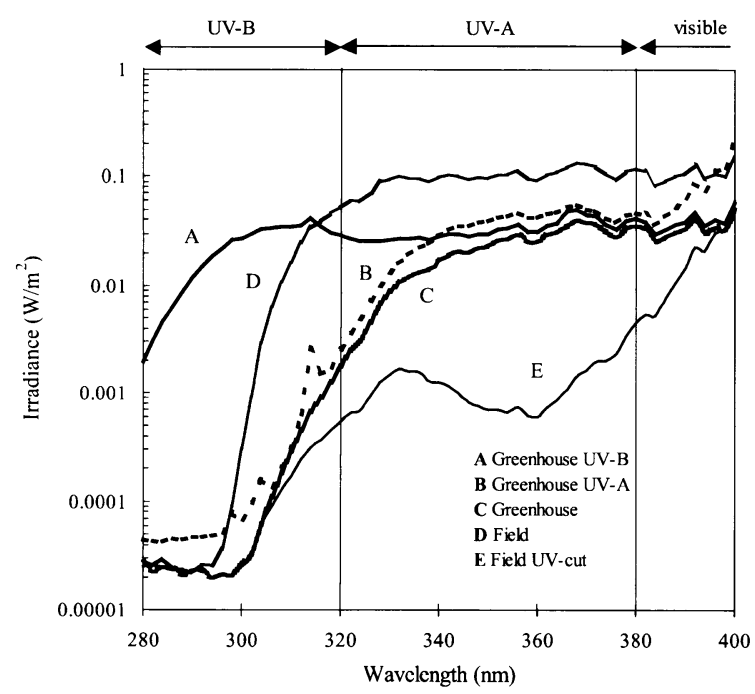

Fig. 1 Irradiance of wavelength from $280 \mathrm{~nm}$ to $400 \mathrm{~nm}$ was measured with a spectroradiometer. The measurements were counted every $2 \mathrm{~nm}$ at the wavelength of $280^{-}-320 \mathrm{~nm}, 320^{-}-380 \mathrm{~nm}$, and $380^{-}-700 \mathrm{~nm}$ correspond to UV-B, UV-A, and visible light, respectively.

UV-cut film under field conditions almost eliminated and diminished the radiation of natural UV radiation $(<380 \mathrm{~nm})$ to less than $3 \%$.

To determine the temporal buildup of anthocyanins in the rose petals, anthocyanins were extracted from each unfolding flower stage of 'Charleston' and 'Ehigasa' grown in the field and analyzed by HPLC. The results of time-course analysis showed that the anthocyanin accumulation started at stage 3 and continued through the anthesis (Fig. 2). It was observed that the accumulation of anthocyanins started by a gradual increase of cyanin, followed by a dramatically increase of chrysanthemin. As a result, in the latter stages of flower development, the petals contained a large amount of chrysanthemin than cyanin. Comparing both cultivars, 'Charleston' is more sensitive to light for accumulation of anthocyanins than 'Ehigasa'. Although the top of the flower bud at stage 2 appeared in red color, no detectable anthocyanin in the enclosed petals with the folded sepals was found in both cultivars.

To obtain more detail information about the UV radiation on flower color changes during the 


\section{ANTHOCYANIN BIOSYNTHESIS IN ROSE}

anthesis, the 'light switching experiment' was carried out (Fig. 3). Field grown rose plants were exposed to UV by covering single bud or flower with UV-cut film through different periods during the anthesis and anthocyanin content in petals of the full opened flower was measured after stage 5. In treatment $\mathrm{A}$ and $\mathrm{C}$, the latter half and the former half of the anthesis was exposed to UV, re-

(a) cv. 'Charleston'

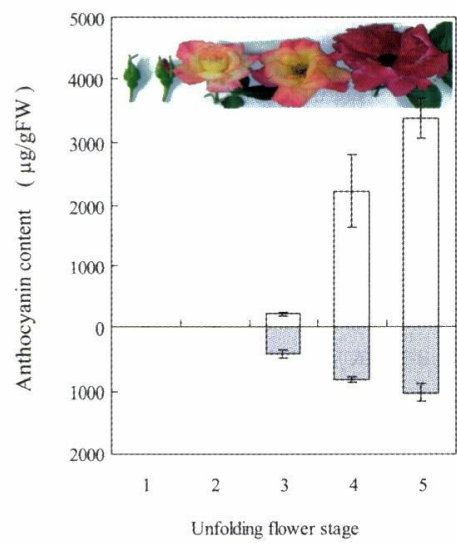

(b) cv. 'Ehigasa'

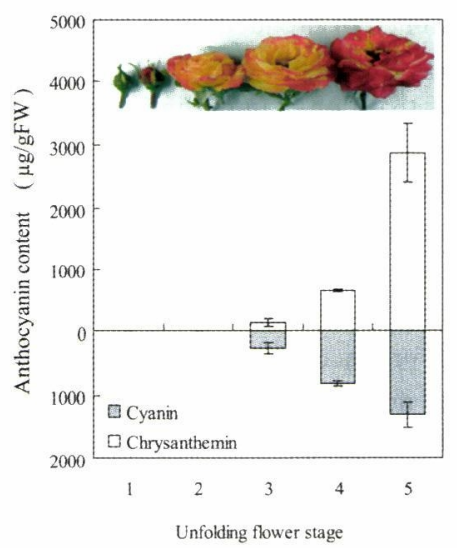

Fig. 2 Anthocyanin accumulation in unfolding flower stages of field-grown rose plants (a) 'Charleston' and (b) 'Ehigasa' under full sunlight. Five different stages were defined as follows: Stage 1, closed bud ( 3 days after flower budding); Stage 2, bud still closed and top of the bud was pigmented (5 days); Stage 3, almost $50 \%$ opened flower and edge of the petal was pigmented ( 8 days); Stage 4 , just opened flower and most petals progress in turning red (10 days); Stage 5, full opened flower before dropout and petals were dark red ( 12 days). The diameter of the full opened flower was about 8 and $10 \mathrm{~cm}$ for 'Charleston' and 'Ehigasa', respectively. Anthocyanin content was shown as the average \pm standard error of at least 10 different flowers.

(a) cv. 'Charleston'

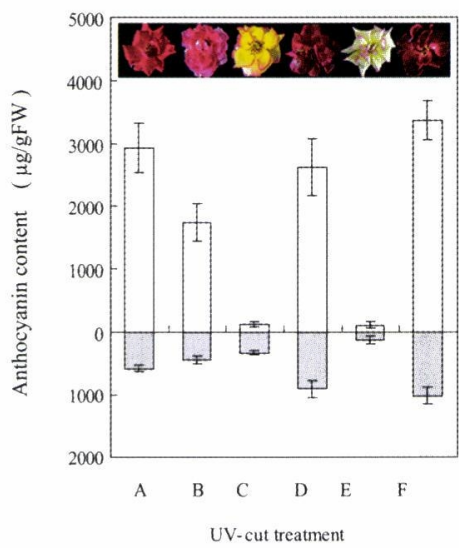

(b) cv. 'Ehigasa'

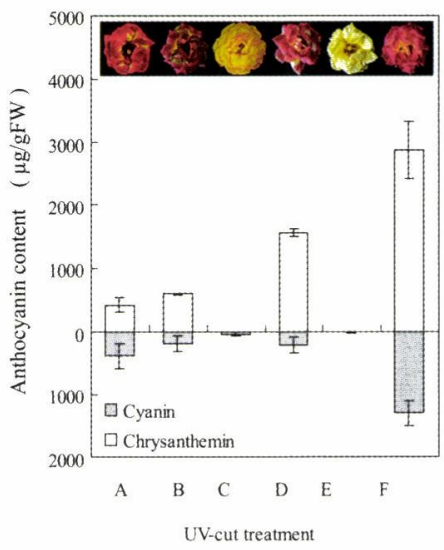

Fig. 3 Effects of different UV-cut treatments (A to F, see in the MATERIALS AND METHODS) on accumulation of anthocyanins in the unfolded petals of field-grown rose plants (a) 'Charleston' and (b) 'Ehigasa'. UV-cut film (Murasaki-A) was used to cover the single bud or flower individually through different unfolding stages. Data was shown as the average \pm standard error of at least 10 different flowers. 


\section{K. HENNAYAKE ET AL.}

spectively, whereas in treatment $B$ and $D$, the unfolding flower stages 4-5 and 1-4 was exposed to $\mathrm{UV}$, respectively. In treatment $\mathrm{B}, \mathrm{UV}$ exposure at latter part of the anthesis produced anthocyanins whereas in treatment $\mathrm{C}$, UV receiving at early stages (about 50\% anthesis) dose not significantly affected on anthocyanin production. Our interpretation of this result is that flower developmental stage is a critical determinant of anthocyanin biosynthesis. In treatment $\mathrm{D}$, only edge of petals was colored in the UV-exposed period until stage 3, then most parts of petals started redding and their color changes were getting on thereafter even under no UV conditions. In contrast, treatment $\mathrm{C}$ completely inhibited or produced very little amount of anthocyanins. The difference between treatment $C$ and $D$ is that petals were exposed to UV until stage 4 at treatment $D$. Therefore, this observation shows that flower unfolding stage 3 to 4 was critical for anthocyanin biosynthesis in rose. This is further confirmed by treatment A where the petals remained yellow throughout the filmcovered period until stage 3 and the color change was rapid after the film was removed. The facts that the accumulation of anthocyanin starts after stage 3 is rather interesting, because it is coincidence with the timing of petals begin to open and lie vertically against the light ray. The negative control treatment $\mathrm{E}$ inhibited or produced very little amount of anthocyanins and the positive control treatment $\mathrm{F}$ produced the maximum amount of anthocyanins. Taken together all these results, we were able to demonstrate that the unfolding flower stage 3 to 4 plays an important role in triggering anthocyanin biosynthesis in rose petals.

We identified the production of anthocyanins, namely chrysanthemin and cyanin in unfolded rose petals and this is consistent with previous studies that report the accumulation of the anthocyanins in plants absorbing UV radiation (Beggs et al., 1986). This prompted an analysis of the effect of UV-A and UV-B radiation on the induction of chrysanthemin and cyanin production in greenhouse conditions. Figure 4 (a) and (b) indicate that both UV-A and UV-B radiation rapidly induce anthocyanin in greenhouse grown 'Charleston'. These results were further confirmed by Fig. 3 treatment $\mathrm{E}$ that inhibited or produced very little amount of anthocyanins. However, Fig. 1 shows that treatment E received very little amount of UV-A radiation (shown in log scale), but it was not enough for mass production. These results reveal that $U V$ radiation enhanced biosynthesis of anthocyanins and the observation that more increase in response to UV-B than UV-A led us to hypothesize that UV-B is necessary to obtain higher resolution of flower color than UV-A radiation. Therefore, it is important to use of artificial supplemental lighting in the actual greenhouse

(a) UV-A supplemental light

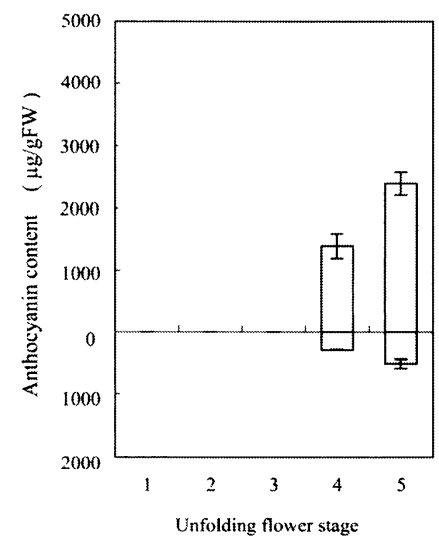

(b) UV-B supplemental light

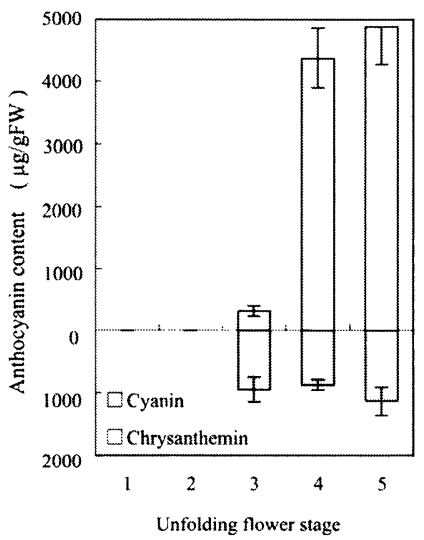

Fig. 4 Anthocyanin accumulation during the unfolding flower stages of rose cultivar 'Charleston', grown in a greenhouse under supplemental lighting with (a) UV-A and (b) UV-B fluorescent lamps. Data was shown as the average \pm standard error of at least 10 different flowers. 
cultivation of rose. In this study, we demonstrated that what kind of lamp to be used and light switching timing (UV turn on/off) to improve the flower quality of green house grown rose. The difference in anthocyanin content may indicate that UV-B radiation stimulates accumulation of higher level of anthocyanin as a protective response in the cells to the damaging effects of UV-B. Although short-wavelength UV-A synthesizes anthocyanins to some extent, it is not harmful for plant and the primary function may be to repair most UV-B-induced DNA damage resulting in accumulation of fewer amounts of anthocyanins.

The anthocyanin biosynthesis pathway is well established (Mol et al., 1989; Forkmann, 1991). The latter stage of the pathway converts leucoanthocyanidin to anthocyanidin, which are first glycosylated at the 3-position in most plants, and these glycosides serve as the substrates for further modification such as glycosylation at the 5-position (Heller and Forkmann, 1988). Anthocyanidin 3-O-glucosyltransferase (3GT) catalyzes the transfer of the glucosyl moiety from UDP-glucose to the 3-hydroxyl group of anthocyanidin, forming anthocyanidin 3-glucoside and anthocyanidin 5-Oglucosyltransferase (5GT) then catalyzes glucosylation of the 5-hydroxyl group of anthocyanidin 3glucoside, forming anthocyanidin 3,5-diglucoside (Mohr and Drumm-Herrel, 1983; Forkmann, 1991). In this study, we suggest that in the latter stages of anthocyanin biosynthesis pathway, ANS, 3GT and 5GT are enhanced by UV-B radiation for flower pigmentation in both cultivars.

Furthermore, the color change does not occur during the absence of UV radiation and the sequence of the anthocyanin biosynthesis is believed to be still progressing to some extent, possibly up to dihydroflavonol in the UV-cut film covered period. Therefore, it is highly expected that a large amount of dihydroflavonols and/or leucoanthocyanidins are accumulated in the petals that are known to be colorless, waiting to be converted into colored anthocyanidin. While, leaf photosynthesis continues to produce and to export glucose by receiving visible light in this period. Consequently, when the film is removed and the flower starts receiving UV radiation, 3GT is probably activated by UV-B and the pathway swiftly flows toward downstream of the biosynthesis ending up with the massive production of chrysanthemin. On the contrary, 5GT activity remains at low level that is not enough to convert all chrysanthemin to cyanin. However, advanced investigation, such as expression and the enzymatic analysis are needed to reveal further details on the regulation of $3 \mathrm{GT}$ and $5 \mathrm{GT}$ by UV-B to understand our knowledge on anthocyanin biosynthesis pathway. To this end, we have already cloned and characterized 3GT and 5GT and further work is in progress.

\section{REFERENCES}

Allen, D. J., Noguěs, S., Morison, J. I. L, Greenslade, P. D., Mcleod, A. R., Baker., N. R. 1999. A thirty percent increase in UV-B has no impact on photosynthesis in well-watered and drought pea plants in the field. Global Change Biol. 5: 235-244.

Arakawa, O., Hori, Y., Ogata, R. 1985. Relative effectiveness and interaction of ultraviolet-B, red and blue light in anthocyanin synthesis of apple fruit. Physiol. Plant. 64: 323-327.

Beggs, C. J., Schnelder-Zlebert, U., Wellmann, E. 1986. UV-B radiation and adaptive mechanisms in plants. In "Stratospheric Ozone Reduction, Solar Ultraviolet Radiation and Plant Life" (ed. by Worrest, R. C., Caldwell, M. M.), Springer-Verlag, New York, p 235-250.

Biolley, J. P., Jay, M., Viricel, M. R. 1994. Flavonoid diversity and metabolism in 100 Rosa hybrida cultivars. Phytochemistry 35: 413-419.

Björn, L. O. 1994. Introduction. In "Photomorphogenesis in Plants" (ed. by Kendrick, R. E., Kronenberg, G. H. M.), Ed. 2, Kluwer Academic Publishers, Boston, p 3-25.

Brugliera, F., Barri-Rewell, G., Holton, T. A., Mason, J. G. 1999. Isolation and characterization of a flavonoid 3'-hydroxylase cDNA clone corresponding to the Htl locus of Petunia hybrida. Plant J. 19: 441451 . 


\section{K. HENNAYAKE ET AL.}

Forkmann, G. 1991. Flavonoids as flower pigments: The formation of the natural spectrum and its extension by genetic engineering. Plant Breed. 106: 1-26.

Gantet, P., Memelink, J. 2002. Transcription factors: tools to engineer the production of pharmacologically active plant metabolites. Trends Pharmacol. Sci. 23: 563-569.

Graham, T. L. 1991. A rapid, high resolution high performance liquid chromatography profiling procedures for plant and microbial aromatic secondary metabolites. Plant Physiol. 95: 584-593.

Harborne, J. B., Grayer, R. J. 1988. The anthocyanins. In "The Flavonoids, Advances in Research Since 1980" (ed. by Harborne, J. B.), Chapman and Hall, London, p 1-20.

Hashimoto, T., Ito, S., Yatsuhashi, H. 1984. Ultraviolet light-induced coiling and curvature of broom sorghum first internodes. Physiol. Plant. 61: 1-7.

Heller, W., Forkmann, G. 1988. Biosynthesis. In "The Flavonoids, Advances in Research Since 1980" (ed. by Harbone, J. B.), Chapman and Hall, London, p 399-425.

Hirner, A. A., Veit, S., Seitz, H. U. 2001. Regulation of anthocyanin biosynthesis in UV-A-irradiated cell cultures of carrot and in organs of intact carrot plants. Plant Sci. 161: 315-322.

Jordan, B. R. 1996. The effects of ultraviolet-B radiation on plants: a molecular perspective. Adv. Bot. Res. 22: 97-162.

Kerr, J. B., McElroy, C. T. 1993. Evidence for large upward trends of ultraviolet-B radiation linked to ozone depletion. Science 262: 1032-1034.

Kochevar, I. E. 1990. UV-induced protein alterations and lipid oxidation in erythrocyte membranes. Photochem. Photobiol. 52: 795-800.

Kolb, C. A., Käser, M. A., Kopeckỳ J., Zotz, G., Riederer, M., Pfündel, E. E. 2001. Effects of natural intensities of visible and ultraviolet radiation on epidermal ultraviolet screening and photosynthesis in Grape leaves. Plant Physiol. 127: 863-875.

Lavola, A., Aphalo, P. J., Lahti, M., Julkunen-Tiitto, R. 2003. Nutrient availability and the effect of increasing UV-B radiation on secondary plant compounds in Scots pine. Environ. Experimen. Bot. 49: 49-60.

Li, J., Ou-Lee, T. M., Raba, R., Amundson, R. G., Last, R. L. 1993. Arabidopsis flavonoid mutants are hypersensitive to UV-B irradiation. Plant Cell 5: 171-179.

Mohr, H., Drumm-Herrel, H. 1983. Coaction between phytochrome and blue/UV light in anthocyanin synthesis in seedlings. Physiol. Plant. 58: 408-414.

Mol, J., Stuitje, A., Gerats, A., Van der Krol, A., Jorgensen, R. 1989. Saying it with genes: Molecular flower breeding. Trends Biotechnol. 7: 148-153.

Robberecht, R., Caldwell, M. M. 1983. Protective mechanisms and acclimation to solar ultraviolet-B radiation in Oenothera stricta. Plant Cell Environ. 6: 477-485.

Shisa, M., Takano, T. 1964. Effects of temperature and light on the coloration of rose flowers. J. Jpn. Soc. Hortic. Sci. 33: 140-146.

Yatsuhashi, H., Hashimoto, T. 1985. Multiplicative action of a UV-B photoreceptor and phytochrome in anthocyanin synthesis. Photochem. Photobiol. 41: 673-680.

Yoshida, K., Mori, M., Kawachi, M., Okuno, R., Kameda, K., Kondo, T. 2003. UV-B resistant polyacylated anthocyanin, HBA, from blue petals of morning glory. Tetrahedron Lett. 44: 7875-7880. 